\title{
Long term refractive outcome in eyes of preterm infants with and without retinopathy of prematurity: comparison of keratometric value, axial length, anterior chamber depth, and lens thickness
}

\author{
Mi Young Choi, In Ki Park, Young Suk Yu
}

\begin{abstract}
Backgroundlaims-A longitudinal study of premature infants was conducted to examine changes in refractive status and their relation with age and factors influencing the occurrence and degree of myopia. Identification of which of the various refractive factors play important parts in relation to myopia in premature infants was attempted.
\end{abstract}

Methods-Under observation were 125 eyes in 65 patients who were found to demonstrate no signs of retinopathy of prematurity (ROP) or who had grade I or II ROP without or after cryotherapy. Cycloplegic refractions were conducted at 6 months, 3 years, and 6 years of age; at 6 years of age keratometric values, lens thicknesses, and axial lengths were recorded, and anterior chamber depths also were measured.

Results-Myopia begins to appear at 6 months of age and its severity increases between the ages of 6 months and 3 years. The condition showed no further progress in subjects older than 3 years. Of the 104 eyes with ROP, those eyes with cicatricial retinopathy tended towards myopia and high myopia while there was no difference in the degree of myopia related to whether or not cryotherapy was conducted. At 6 years of age, the premature infants exhibited shallower anterior chambers, thicker lenses, and higher axial lengths when the degree of the myopia was higher. The keratometric values, however, appeared to bear no relation to the degree of the myopia.

Conclusion-These results suggest that the occurrence of myopia is related more strongly to whether or not there is cicatricial retinopathy than whether or not there is cryotherapy. Also, the degree of the myopia was found to be related to the depth of the anterior chamber, the thickness of the lens, and the change in axial length but not to keratometric value. (Br F Ophthalmol 2000;84:138-143)

Correspondence to: Young Suk Yu, MD, Department of Ophthalmology, College of Medicine, Seoul National University, 28 Yongon-dong, Chongro-gu, Seoul 110-744, Korea

Accepted for publication 15 September 1999
Although a few studies reported that there was no difference in the prevalence of myopia in children born prematurely, ${ }^{2-5}$ it is now con- firmed that myopia of prematurity without previous retinopathy of prematurity (ROP) does occur, and at higher frequencies than in the full term babies. ${ }^{6}{ }^{7}$ Moreover, eyes demonstrating ROP continue to exhibit signs of myopia, and the degree and frequency of myopia occurrence is known to be related to the status of the ROP. ${ }^{8-11}$

The mechanisms of myopia in premature infants are not clearly understood. Of the various factors that influence refractive status, those related to myopia in prematurely born babies are believed to include an increase in keratometric value, ${ }^{12}{ }^{13}$ a decrease in the depth of the anterior chamber, ${ }^{14}{ }^{15}$ and high refractive power of the lens. ${ }^{16}$ Our survey of the existing literature, however, reveals that an analysis of the refractive elements related to final refractive values has not yet been conducted using a longitudinal study of the refractive status of premature infants.

The present study was conducted to examine changes in refractive status and their relation with age over the long term in premature Korean infants and to identify those refractive elements that have an effect on the occurrence, frequency, and degree of myopia in these infants.

Subjects and methods

For the diagnosis of ROP, fundus examinations were performed by a paediatric ophthalmologist (YSY). Examinations were conducted between 4 and 6 weeks of chronological age in infants with birth weights of $1500 \mathrm{~g}$ or less or with a gestational age of 28 weeks or less. Informed consent was obtained from the parents of all participants before testing. The nature of the follow up examinations depended on the findings made at the first examination. Infants with no ROP were examined at 2-4 week intervals until vascularisation proceeded into zone III. Infants with ROP were examined at least every 1-2 weeks. On infants with disease defined as threshold by the criteria set forth by the Multicenter Trial of Cryotherapy for Retinopathy of Prematurity, ${ }^{17}$ cryotherapy was performed within 72 hours of diagnosis. The active stages of ROP were classified according to the International Classification of Retinopathy of Prematurity ${ }^{18}$ and the cicatricial stages were divided according to Reese's classification. ${ }^{19}$

Of the premature infants who visited the department of paediatric ophthalmology at Seoul National University Hospital from Feb- 
Table 1 Cycloplegic refractions at 6 months, 3 years, and 6 years of age as related to status of retinopathy of prematurity (ROP). Spherical equivalent (mean (SD))

\begin{tabular}{lllllll}
\hline & Group R-0 (21 eyes) & p Value (t test) & Group R-1 (75 eyes) & p Value (t test) & Group R-2 (29 eyes) & Total (125 eyes) \\
\hline 6 Months & $0.22(2.69)$ & 0.000 & $-2.37(3.65)^{\star}$ & 0.006 & $-5.16(4.64)$ & $-2.58(4.11)$ \\
3 Years & $0.11(2.21)$ & 0.000 & $-3.55(4.47)^{\star}$ & 0.160 & $-5.13(5.26)$ & $-3.30(4.67)$ \\
6 Years & $0.27(1.46)$ & 0.000 & $-3.54(4.37)$ & 0.510 & $-4.19(4.50)$ & $-3.05(4.32)$ \\
\hline
\end{tabular}

${ }^{\star} \mathrm{p}$ Value $=0.000$ (paired $t$ test)

Group R-0=no ROP; group R-1=no cicatricial ROP with a history of ROP, regardless of treatment; group R-2=cicatricial ROP, grade II.

ruary 1987 to April 1993, those who did not show signs of ROP or who were classified as being in the no cicatricial change or the cicatricial grade I or II of ROP without or after treatment for acute ROP were tested. All subjects were available for observation at 6 months, 3 years, and 6 years of age. A cycloplegic refraction was conducted on subjects at 6 months and 3 years of age, and a cycloplegic refraction and measurement of the keratometric value, the depth of the anterior chamber, the thickness of the lens, and the axial length (measured using ultrasonic waves) were conducted on subjects at 6 years of age. The cycloplegic refraction was conducted with instillation of $2.5 \%$ phenylephrine and $1 \%$ tropicamide three times at 5 minute intervals. When cycloplegia and dilatation of the pupils were sufficient, the test was conducted with retinoscopy. All patients aged 6 months and those aged 3 years who were uncooperative were sedated with chloral hydrate. All patients aged 6 years were awake during the test. The refractive value was recorded as a spherical equivalent, and myopia was defined as high when the spherical equivalent was equal to or higher than -6.0 dioptres.

Patients were classified into three groups: the R-0 group (no ROP) comprised those patients

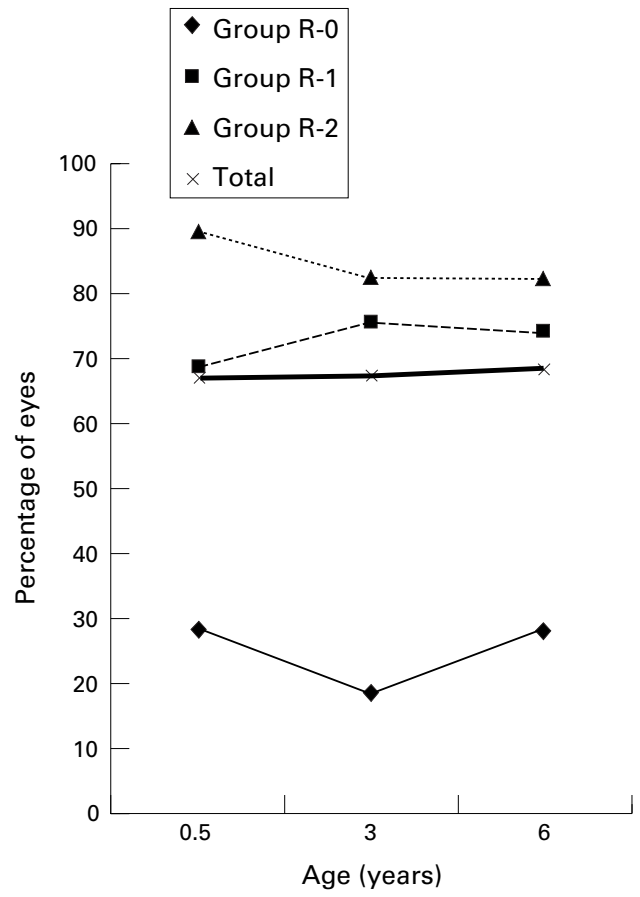

Figure 1 Prevalence of myopia at 6 months, 3 years, and 6 years of age as related to the presence of retinopathy of prematurity (ROP). Group R-0: no ROP; group R-1: no cicatricial $R O P$ with a history of $R O P$, regardless of treatment; group R-2: cicatricial ROP, grade II. with no history of ROP; the R-1 group (no cicatricial ROP) included patients with a history of ROP but whose condition had completely disappeared without leaving cicatricial retinopathy, regardless of treatment; and the R-2 group (cicatricial ROP) was made up of patients who were currently demonstrating ROP of the cicatricial II phase - that is, fundus appearance of macular dragging. Changes in refractive status and the frequency of myopia and high myopia were recorded for each group. Using the spherical equivalents of 6 year olds as the criteria, eyes were classified into the following three groups: $\mathrm{M}-0$ (no myopia) consisted of eyes with emmetropia or hyperopia; M-1 (no high myopia) comprised eyes of below -6.0 dioptres myopia; and M-2 (high myopia) included eyes with myopia at a degree equal to or higher than -6.0 dioptres. Changes in the refractive status were checked in each group over time, and the ratio of each group was recorded in accordance with cryotherapy. The spherical equivalents of 6 month olds and 6 year olds were compared, while the spherical equivalent, keratometric value, axial length, depth of anterior chamber, and thickness of lens of 6 year olds in each group were compared. Statistical analysis was set at $t$ test and paired $t$ test.

\section{Results}

A total of 125 eyes of 65 patients were checked-54 eyes of 28 males and 71 eyes of 37 females. Cycloplegic refraction was conducted on all eyes at 6 months, 3 years and 6 years of age, while keratometric value and axial length were measured in 6 year olds only. Of the total, 103 eyes were examined for anterior chamber depth and lens thickness at 6 years of age.

In accordance with the ROP based classification, 21 eyes were included in the R-0 group, and the remaining 104 eyes exhibited ROP (ROP stage 1 in five eyes, ROP stage 2 in 14 eyes, ROP stage 3 in 83 eyes, and ROP stage $4 \mathrm{~A}$ in two eyes). Of 104 eyes, 75 eyes were placed in the R-1 group (patients with a history of ROP but whose condition had completely disappeared without leaving cicatricial retinopathy, regardless of treatment), and 29 eyes were classified into the R-2 group (patients with ROP of cicatricial grade II). In 12 eyes $(41.4 \%)$ in the $\mathrm{R}-2$ group, the cicatricial change of the retina occurred spontaneously and without treatment for the ROP. The remaining 17 eyes $(58.6 \%)$ in the $\mathrm{R}-2$ group exhibited cicatrical retinopathy after cryotherapy. Seventeen $(25.4 \%)$ of the 67 eyes with cryotherapy showed cicatricial change of the retina. 


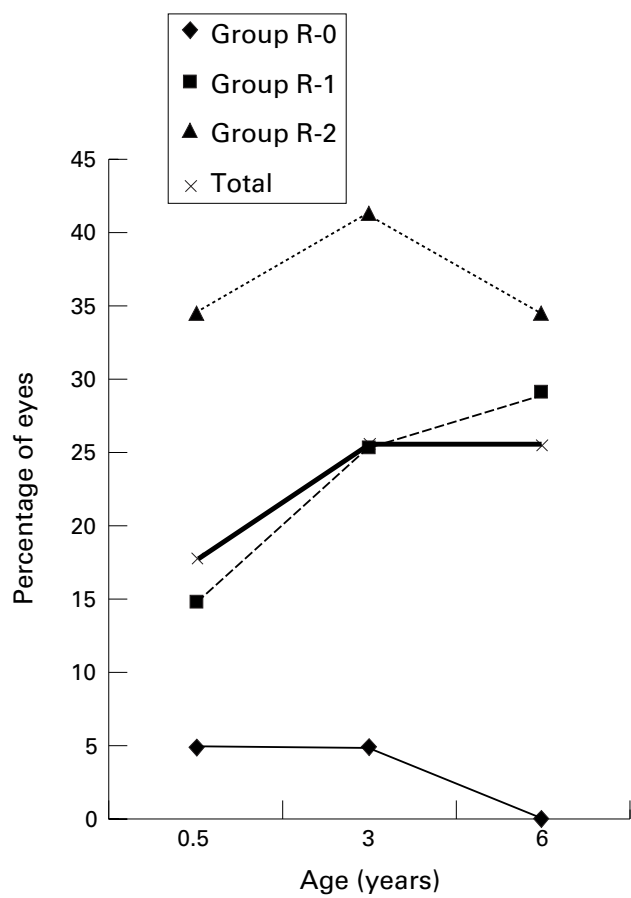

Figure 2 Prevalence of high myopia at 6 months, 3 years, and 6 years as related to the presence of retinopathy of prematurity (ROP). Group R-0: no ROP; group R-1: no cicatricial ROP with a history of $R O P$, regardless of treatment; group $R-2$ : cicatricial ROP, grade II.

An analysis comparing changes in refractive status with age in each group (Table 1) revealed no changes in the R-0 and R-2 groups, but in the R-1 group the degree of myopia increased between 6 months and 3 years of age, with no change found from 3 years to 6 years. The R-1 group displayed more severe myopia $(p<0.005)$ than the $\mathrm{R}-0$ group during the period between 6 months and 3 years of age, while the R-2 group showed more severe myopia than the R-1 group at 6 months, 3 years, and 6 years of age. The difference was statistically significant only at 6 months $(p=0.006)$, however. The frequency of myopia and high myopia increased in ascending order through groups R-0, R-1, and R-2. An analysis relating the frequency of myopia and high myopia with age in each group is shown in Figures 1 and 2 . There was no relation found between the rate of myopia and age in any of the groups, and the rate of high myopia varied across the groups. In 3 year olds and 6 year olds in the R-0 group, high myopia had disappeared, and in the R-1 group, the rate of high myopia approximately doubled. In the R-2 group, high myopia occurred at a high rate at 6 months of age and was found present at 6 years of age.

According to the classification based on the spherical equivalent determined through a cycloplegic refraction conducted at 6 years of

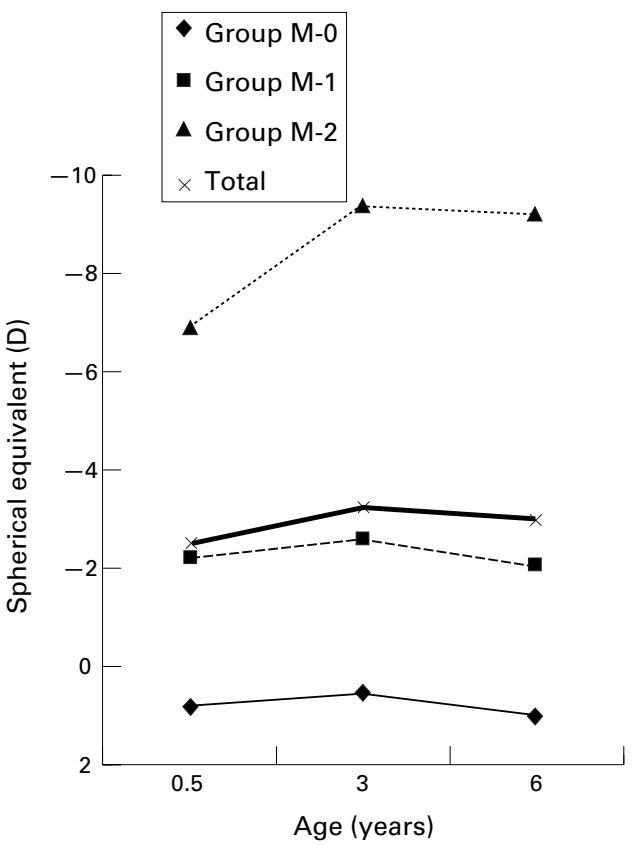

Figure 3 Changes of spherical equivalents in cycloplegic refraction as related to refractive status at 6 years of age. Group M-0: emmetropia or hyperopia; group $M-1$. myopia $(<-6.0 \mathrm{D})$; group $M-2$ : high myopia $(\geqslant-6.0 \mathrm{D})$.

age, 36 eyes were classified into the $\mathrm{M}-0$ group (eyes with emmetropia or hyperopia), 57 eyes were classified into the $M-1$ group (eyes with myopia below -6.0 dioptres), and 32 eyes were classified into the M-2 group (eyes with myopia above -6.0 dioptres). An analysis comparing refractive status with age in each group showed no age related change of refractive status in the $M-0$ and $M-1$ groups (Fig 3). In the M-2 group, the degree of myopia increased with statistical significance $(p=0.000)$ between 6 months and 3 years of age, while there was no change between 3 years and 6 years of age $(p=$ 0.532 ). The degree of myopia was higher with statistical significance $(p=0.000$, Table 2) in eyes treated with cryotherapy than in eyes that did not receive treatment.

In 6 year olds, analysis of the degree of myopia, keratometric value, anterior chamber depth, lens thickness, and axial length revealed no statistically significant correlation between myopia and keratometric value. Axial length, however, was found to increase with statistical significance with the degree of myopia ( $p=$ 0.000 ). The depth of the anterior chamber in the M-2 group (high myopia) was less than in the other two groups and the difference was statistically significant $(p=0.001)$. Lens thickness correlated positively with the degree of myopia but the relation was not statistically significant $(p=0.028$, Table 3$)$.

Table 2 Refractive status at 6 years of age according to cryotherapy (eyes (\%))

\begin{tabular}{llllll}
\hline & CR at 6 years (mean (SD)) & Group M-0 (36 eyes) & Group $M-1$ (57 eyes) & Group M-2 (32 eyes) & Total (125 eyes) \\
\hline Cryotherapy (-) & $-1.38(3.44)^{\star}$ & $28(48.3)$ & $24(41.4)$ & $6(10.3)$ & $58(100)$ \\
Cryotherapy (+) & $-4.30(4.49)^{\star}$ & $8(11.9)$ & $33(49.3)$ & $26(38.8)$ & $67(100)$ \\
\hline
\end{tabular}

${ }^{\star} \mathrm{p}$ Value $=0.000(t$ test $)$

$\mathrm{CR}=$ cycloplegic refraction.

Group $M-0=$ emmetropia or hyperopia; group $M-1=$ myopia $(<-6.0 \mathrm{D})$; group $\mathrm{M}-2=$ high myopia $(\geqslant-6.0 \mathrm{D})$. 
Table 3 Cycloplegic refraction at 6 months and 6 years of age, and keratometric value, axial length, anterior chamber depth and lens thickness at 6 years as related to refractive status at 6 years of age (mean (SD))

\begin{tabular}{|c|c|c|c|c|c|c|}
\hline \multirow[b]{2}{*}{ Group } & \multirow{2}{*}{$\begin{array}{l}6 \text { Months } \\
\text { CR (D) } 125 \text { eyes }\end{array}$} & \multicolumn{5}{|l|}{6 Years } \\
\hline & & CR (D) 125 eyes & $K$ (D) 125 eyes & $\begin{array}{l}A X L(\mathrm{~mm}) \\
125 \text { eyes }\end{array}$ & $\begin{array}{l}A C D(\mathrm{~mm}) \\
103 \text { eyes }\end{array}$ & $\begin{array}{l}L T(\mathrm{~mm}) \\
103 \text { eyes }\end{array}$ \\
\hline M-0 (36 eyes) & $0.76(2.08)$ & $0.94(0.89)$ & $44.49(1.71)$ & $21.85(0.88)$ & $3.23(0.29)$ & $3.82(0.32)$ \\
\hline M-1 (57 eyes) & $-2.23(2.22)$ & $-2.12(1.90)$ & $44.23(1.44)$ & 22.56 & $3.15(0.41)$ & $3.91(0.31)$ \\
\hline M-2 (32 eyes) & $-6.97(4.56)$ & $-9.19(2.87)$ & $44.42(1.94)$ & $24.62(1.39)$ & $2.97(0.30)$ & $4.01(0.29)$ \\
\hline \multicolumn{7}{|l|}{$\mathrm{p}$ Value ( $t$ test) } \\
\hline$M-0$ and $M-1$ & 0.000 & 0.000 & 0.415 & 0.002 & 0.328 & 0.233 \\
\hline$M-1$ and $M-2$ & 0.000 & 0.000 & 0.606 & 0.000 & 0.049 & 0.190 \\
\hline$M-0$ and $M-2$ & 0.000 & 0.000 & 0.852 & 0.000 & 0.001 & 0.028 \\
\hline
\end{tabular}

Group $M-0=$ emmetropia or hyperopia; group $M-1=$ myopia $(<-6.0 \mathrm{D})$; group $\mathrm{M}-2=$ high myopia $(\geqslant-6.0 \mathrm{D})$.

$\mathrm{CR}=$ cycloplegic refraction; $\mathrm{K}=$ keratometric value; $\mathrm{AXL}=$ axial length; $\mathrm{ACD}=$ anterior chamber depth; $\mathrm{LT}=$ lens thickness .

\section{Discussion}

There have been many studies on the occurrence of myopia in prematurely born babies. In cases where the severity of the ROP required treatment with cryotherapy or where there was cicatricial retinopathy, the frequency and degree of the myopia increased. It is generally agreed that the degree and frequency of myopia are proportional to changes in cicatricial retinopathy, ${ }^{10}{ }^{11} 20-22$ but there remains some controversy over the refractive abnormalities in eyes in which the ROP disappeared spontaneously. While Schaffer et $a l^{23}$ and Kim et al ${ }^{20}$ report finding the degree and frequency of myopia in these cases to be similar to those in eyes without ROP, Fledelius ${ }^{6}$ and Holmström et $a l^{7}$ maintain that the frequency of myopia was high in premature infants regardless of ROP.

In the present study, $84(67.2 \%)$ of 125 eyes were found to be myopic when their refractive status was studied. Specifically, eyes with high myopia accounted for 22 , or $26.2 \%$, cases of myopia, or $17.4 \%$ of the total eyes. According to other reports on the frequency of myopia related to prematurity, ${ }^{64-26}$ the frequency of myopia at 5 years of age varied between 5\% and $28.5 \%$ and that of high myopia ranged between $2.3 \%$ and $9.7 \%$. One possible reason for the high frequency of myopia and high myopia in the present study may be related to the retinal status of the patients and the cryotherapy. Firstly, eyes with ROP comprised $83.2 \%$ (104 of 125 eyes) of the total. Secondly, eyes treated with cryotherapy made up 53.6\% ( 67 of 125 eyes) of the total. Thirdly, eyes with cicatricial retinopathy accounted for $23.2 \%$ ( 29 of 125 eyes) of the total. Of the 42 eyes not treated with cryotherapy and not accompanied by cicatricial retinopathy, $38.1 \%$ (16 eyes) demonstrated myopia and $2.4 \%$ (one eye) exhibited high myopia.

Of the 21 eyes without ROP, 19.0\% (four eyes) displayed myopia without high myopia. Eyes with ROP, particularly those left with cicatricial retinopathy, showed a high frequency of myopia and high myopia. These findings are compatible with previous results.

An analysis of refractive status in 6 year olds revealed that age related changes in refractive status were similar in all the eyes. Shapiro et $\mathrm{al}^{4}$ have reported finding no refractive change related to prematurity in subjects without ROP ranging in age from 6 months to $31 / 2$ years. In contrast, Quinn et $a l^{8}$ have reported finding that the refractive status in prematurely born infants changed to myopia between the ages of 3 months and 1 year but did not change thereafter. Eyes with a high degree of myopia in 6 year olds were found to have already attained that high degree by 6 months of age. This corresponds to the study by Quinn et $a l_{,}^{8}$ which reports that the existence of myopia and high myopia in $5^{1 / 2}$ year olds can be predicted based on the degree of myopia at 3 months of age in premature infants. The degree of myopia increased with statistical significance between the ages of 6 months and 3 years only in the M-2 group (high myopia). Changes in the severity of the myopia were not found between the ages of 3 years and 6 years. Based on these findings, we can infer that the refractive status of eyes without ROP stabilises at a relatively early stage, but eyes with ROP, particularly cases involving cryotherapy treatment, are susceptible to dramatic changes in refractive status after 6 months of age.

There is some debate about the influence of cryotherapy on prematurity related myopia. Ben-Sira $e t ~ a l^{27}$ report finding the degree of myopia to be higher in eyes with cryotherapy and Laws $e t$ al $^{28}$ maintain that myopia is more likely to occur with cryotherapy than with laser treatment. In contrast, Kim et al ${ }^{20}$ report observing that although cryotherapy increased the frequency of myopia, it did not intensify the degree. According to studies by Nissenkorn $e t$ $a l,{ }^{10}$ no difference in the degree and frequency of myopia was found between eyes that had cicatricial retinopathy without treatment and eyes that had cicatricial retinopathy after cryotherapy, which suggests that the cryotherapy itself did not cause the myopia.

In the present study, the degree and frequency of myopia were higher in eyes treated with cryotherapy than in eyes that were not treated. But in many cases eyes treated with cryotherapy showed a high degree of ROP. Therefore, in order to examine whether it was the cryotherapy or the ROP, or cicatricial retinopathy, that resulted in the myopia, we classified 29 eyes showing cicatricial retinopathy according to the existence of cryotherapy. The average myopia value in 6 year olds was -2.79 dioptres in 17 eyes that had undergone cryotherapy, and -6.18 dioptres in 12 eyes that had not undergone cryotherapy (Table 4). Through this, it was revealed that cryotherapy does not aggravate ROP related 
Table 4 Refractive status at 6 years of age according to cryotherapy in cicatricial retinopathy of prematurity (eyes (\%))

\begin{tabular}{llllll}
\hline & CR at 6 years (mean (SD)) & Group M-0 (36 eyes) & Group M-1 (57 eyes) & Group M-2 (32 eyes) & Total (29 eyes) \\
\hline Cryotherapy (-) & $-6.18(3.91)^{\star}$ & $0(00.0)$ & $7(58.3)$ & $5(41.7)$ & $12(100)$ \\
Cryotherapy (+) & $-2.79(4.46)^{\star}$ & $5(29.4)$ & $7(41.2)$ & $5(29.4)$ & $17(100)$
\end{tabular}

${ }^{\star} \mathrm{p}$ Value $=0.045(t$ test $)$

$\mathrm{CR}=$ cycloplegic refraction

Group $\mathrm{M}-0=$ =mmetropia or hyperopia; group $\mathrm{M}-1=$ myopia $(<-6.0 \mathrm{D})$; group $\mathrm{M}-2=$ high myopia $(\geqslant-6.0 \mathrm{D})$.

myopia and that cicatricial retinopathy is an important factor in the occurrence of myopia.

There is also debate over which of the refractive elements, including keratometric value, depth of anterior chamber, thickness of lens, and axial length, have an influence on the overall refractive status in cases of prematurity related myopia. Yamamoto et al ${ }^{12}$ report that the degree of myopia and the keratometric value increased when the cicatricial retinopathy was severe but that the axial length was not influenced. In a study of 17 patients with a history of ROP but who no longer showed signs of the condition, Gallo and Fagerholm ${ }^{13}$ report finding that the refractive power of the cornea played an important part in the occurrence of myopia. Majima ${ }^{14}$ examined 121 subjects, who were born prematurely and whose weight was less than $1500 \mathrm{~g}$ at the time of birth, when they were aged 5-20 years and reported finding a positive correlation between the severity of the cicatricial retinopathy and the degree of the myopia. Also, according to the study, with more severe cicatricial retinopathy, the depth of the anterior chamber decreased while the thickness of the lens increased but neither the keratometric value nor the axial length was influenced. According to a study by Koraszewska-Matuszewska et al ${ }^{15}$ on 103 patients who weighed between $580 \mathrm{~g}$ and $2450 \mathrm{~g}$ at the time of birth and who began demonstrating myopia when they were aged between 3 and 15 years, myopia is not related to axial length. In contrast, Laws et $a l^{29}$ report finding a negative correlation between the severity of ROP and axial length, suggesting that changes in the axial length are not caused by the ROP but by prematurity. The report in this way supports the work of Gordon and Donzis ${ }^{16}$ who point to high refractive power of the lens as a major cause of myopia.

In the present study, the degree of myopia and the keratometric value in 6 year olds were found to have no relation, but other refractive elements showed corresponding differences. The depth of the anterior chamber was lower with statistical significance $(p=0.001)$ in the M-2 group compared with other groups, and the thickness of the lens in the M-2 group was higher with statistical significance $(p=0.027)$. The axial length increased with statistical significance through, in ascending order, the M-0 group (emmetropia or hyperopia), the M-1 group (myopia below -6.0 dioptres), and the M-2 group (high myopia above -6.0 dioptres). Lee et $a l^{30}$ report the average axial length in emmetropia of 6 year olds to be $22.18 \mathrm{~mm}$. The present study found an average axial length of $21.96 \mathrm{~mm}$ in the M-0 group, 22.74 $\mathrm{mm}$ in the $\mathrm{M}-1$ group, and $24.77 \mathrm{~mm}$ in the M-2 group. Although it is not possible to com- pare these results statistically with those observed by Lee et $a l,{ }^{30}$ it should be noted that the M-2 group yielded a widely varying result.

In conclusion, a decrease in the anterior chamber depth, and an increase in the lens thickness and axial length combine to cause myopia in prematurely born patients while the keratometric value appears not to be an influential factor. According to longitudinal observation of refractive values in prematurely born subjects, prematurity related myopia occurs at a relatively early stage in life, continuing from 6 months of age to 3 years and stabilising thereafter. In cases involving ROP, particularly cicatricial retinopathy, the frequency of myopia and high myopia is high, suggesting that correction of the refractive abnormality is critical.

1 Dobson V, Fulton AB, Manning K. Cycloplegic refractions of premature babies. Am f Ophthalmol 1981;91:490-5.

2 Fletcher MC, Brandon S. Myopia of prematurity. Am $\mathcal{F}$ Ophthalmol 1955;40:474-81.

3 Graham MV, Gray OP. Refraction of premature babies' eyes. BMF 1963;1:1452-4.

4 Shapiro A, Yanko L, Nawratzki I, et al. Refractive power of premature children at infancy and early childhood. $A m \mathcal{F}$ Ophthalmol 1980;90:234-8.

5 Saw S-M, Chew S-J. Myopia in children born premature or with low birth weight. Acta Ophthalmol Scand 1997;75: $548-50$

6 Fledelius HC. Pre-term delivery and subsequent ocular development. A 7-10 year follow-up of children screened 1982-84 for ROP. 3. Refraction. Myopia of prematurity. Acta Ophthalmol Scand 1996;74:297-300.

7 Holmstrkm G, Azazi M, Kugelberg U. Ophthalmological long term follow up of preterm infants: a population based, prospective study of the refraction and its development. $\mathrm{Br}$ f Ophthalmol 1998;82:1265-71.

8 Quinn GE, Dobson V, Kivlin J, et al. Cryotherapy for Retinopathy of Prematurity Cooperative Group. Prevalence of myopia between 3 months and $51 / 2$ years in preterm infants with and without retinopathy of prematurity. Ophthalmology 1998;105:1292-300.

9 Lue CL, Hansen RM, Reisner DS, et al. The course of myopia in children with mild retinopathy of prematurity. Vis pia in children with mill.

10 Nissenkorn I, Yassur Y, Mashkowski D, et al. Myopia in premature babies with and without retinopathy of prematurity. Br F Ophthalmol 1983;67:170-3.

11 Robinson R, O'Keefe M. Follow-up study on premature infants with and without retinopathy of prematurity. $\mathrm{Br} \mathcal{F}$ Ophthalmol 1993;77:91-4.

12 Yamamoto $\mathrm{M}$, Tatsugami $\mathrm{H}$, Bun $\mathrm{J}$. A follow-up study of refractive errors in premature infants. $\mathcal{F p}_{p} \mathcal{f}$ Ophthalmol 1979;23:435-43.

13 Gallo JE, Fagerholm P. Low-grade myopia in children with regressed retinopathy of prematurity. Acta Ophthalmol (Copenh) 1993;71:519-23.

14 Majima A. Studies on retinopathy of prematurity II. Fundus appearance and ocular functions in cicatricial phase of very

15 Koraszewska-Matuszewska B, Samochowiec-Donocik E, Pieczara E, et al. Myopia as a complication of retinopathy Pieczara E, et al. Myopia as a complication
of prematurity. Klin Oczna 1993;95:339-42.

16 Gordon RA, Donzis PB. Myopia associated with retinopathy of prematurity. Ophthalmology 1986;93:1593-8.

17 Cryotherapy for Retinopathy of Prematurity Cooperative Group. Multicenter trial of cryotherapy for retinopathy of prematurity. Preliminary results. Arch Ophthalmol 1988; 106:471-9.

18 Committee for the Classification of Retinopathy of Prematurity. An international classification of retinopathy of prematurity. Arch Ophthalmol 1984;102:1130-4.

19 Reese AB, King MJ, Owens WC. A classification of retrolental fibroplasia. Am f Ophthalmol 1953;36:1333-5.

$20 \mathrm{Kim}$ JY, Kwak SI, Yu YS. Myopia in premature infants at the age of 6 months. Korean 7 Ophthalmol 1992;6:44-9.

21 Khwarg SI, Yu HG, Yu YS. Change of refraction in premature infants after cryotherapy for retinopathy of prematu- 
rity between the age of six months and three years. Korean F Ophthalmol 1995;9:111-6.

22 Laws D, Shaw DE, Robinson J, et al. Retinopathy of prematurity: a prospective study. Review at six months. Eye 1992;6:477-83

23 Schaffer DB, Quinn GE, Johnson L. Sequelae of arrested mild retinopathy of prematurity. Arch Ophthalmol 1984 102:373-6

24 Fledelius HC. Ophthalmic changes from age of 10 to 18 years. A longitudinal study of sequels to low birth weight. 1 . Refraction. Acta Ophthalmol (Copenh) 1980;58:889-98.

25 Cats BP, Tan KE. Prematures with and without regressed retinopathy of prematurity: comparison of long-term $(6-10$
years) ophthalmological morbidity. $₹$ Pediatr Ophthalmol Strabismus 1989;26:271-5.
26 Gallo JE, Lennerstrand G. A population-based study of ocular abnormalities in premature children aged 5 to 10

27 Ben-Sira I, Nissenkorn I, Weinberger D, et al. Long-term results of cryotherapy for active stages of retinopathy of prematurity. Ophthalmology 1986;93:1423-8.

28 Laws F, Laws D, Clark D. Cryotherapy and laser treatment for acute retinopathy of prematurity: Refractive outcome, a longitudinal study. Br f Ophthalmol 1997;81:12-5.

29 Laws DE, Haslett R, Ashby D, et al. Axial length biometry in infants with retinopathy of prematurity. Eye 1994;8:42730.

30 Lee EK, Lee DB, Jin $\mathrm{KH}$, et al. The study of correlation between axial length and refractive error in Korean children. F Korean Ophthalmol Soc 1993;34:654-60. 